\title{
Revisiting Variance Gamma Pricing: An Application to S\&P500 Index Options
}

\section{Sharif Mozumder ${ }^{1}$}

Ghulam Sorwar $^{2}$

Kevin Dowd ${ }^{3}$

May 18, 2015

${ }^{1}$ Corresponding author; Associate Professor of Mathematics, Department of Mathematics, University of Dhaka, Dhaka, Bangladesh. Email: sharif_math2000@yahoo.com.

${ }^{2}$ Professor of Finance, Salford Business School, Lady Hale Building, Salford M5 4WT, United Kingdom. Email: G.Sorwar@Salford.ac.uk. Corresponding author.

${ }^{3}$ Professor of Finance and Economics, Durham Business School, Mill Hill Lane, Durham DH1 3LB, United Kingdom. Email: k.dowd@durham.ac.uk. 
Revisiting Variance Gamma Pricing: An Application to S\&P500 Index Options

\begin{abstract}
We reformulate the Lévy-Kintchine formula to make it suitable for modelling the stochastic time-changing effects of Lévy processes. Using Variance-Gamma (VG) process as an example, it illustrates the dynamic properties of a Lévy process and revisits the earlier work of Geman (2002). It also shows how the model can be calibrated to price options under a Lévy VG process, and calibrates the model on recent S\&P500 index options data. It then compares the pricing performance of Fast Fourier Transform (FFT) and Fractional Fourier Transform (FRFT) approaches to model calibration and investigates the trade-off between calibration performance and required calculation time.
\end{abstract}

Keywords: Variance Gamma process, infinitely divisible distribution, Fast Fourier Transform. 


\section{Introduction}

In the seminal papers by Black and Scholes (BS) (1973) and Merton (1973) the underlying distribution driving the option price is the lognormal distribution, which itself reflects the underlying assumption that returns follow a Geometric Brownian Motion (GBM). However, the use of GBM as the benchmark process for describing asset returns in continuous time does not allow for some key features of stylized asset price behavior: in particular, it fails to allow for (i) asset price jumps, (ii) conditional skewness in returns, (iii) conditional excess kurtosis in returns, (iv) stochastic volatility or (v) any correlation between returns and their volatilities. These well-established features of empirical return process have encouraged researchers to develop alternative models in which GBM has been replaced with more sophisticated processes, including GARCH processes, stochastic volatility processes and Lévy processes, which are the focus of the present paper.

Lévy processes can deal with each of these deficiencies, and therefore have considerable potential for option pricing: (i) Traditionally, jumps in asset prices have been modeled by the compound Poisson process which allows for a finite number of jumps within a finite interval. However, we often observe that asset prices display many small jumps on a fine time scale, and this suggests that Lévy processes which allow infinite numbers of jumps to occur within a finite time scale may be more suitable for modeling jumps. (ii/iii) Lévy processes can easily accommodate both skewness and excess kurtosis in returns. (iv) Lévy processes can accommodate stochastic volatility by applying stochastic time change: this is the equivalent to stochastically altering the clocks on which the Lévy process is run. If we view the original clock as calendar time and the new random clock as the business 
time, a more active business day implies a faster clock. (v) To model the correlation between returns and their volatilities, we let the innovations in the Lévy process be correlated with the innovations in the random clock on which it is run. When this correlation is negative, the clock tends to run faster when the Lévy process falls (Carr and Wu (2002)). In summary, time-changed Lévy processes parsimoniously capture all five main deficiencies of Brownian motion. Furthermore, Lévy processes lead to analytically tractable models which simplifies parameter estimation and model calibration.

In this study we seek to make a number of contributions to the Lévy option pricing literature. First, we reformulate the Lévy-Kintchine formula on which Lévy processes are based: this allows us to explicitly clarify how additional shocks to the Brownian motion dynamics can be regarded as the superpositions of compound Poisson and compensated compound Poisson paths. Our reformulation is helpful for modeling the time-changing effects, aids in both the simulation and parameter estimation of Lévy processes, and has ready applications to Lévy option pricing. Second, we use this reformulation to visit the earlier work of Geman (2002): we are able to demonstrate a misspecification in this work and provide a correct solution to it. Third, using observed option prices based on the S\&P500, we calibrate the BS model and its time-changed Lévy version, the Variance Gamma (VG) model. Fourth, we use two different Fourier techniques to value the options and contrast their performance with each other. The two Fourier techniques considered are the Fast Fourier Transform (FFT) and Fractional Fast Fourier Transform (FRFT) suggested by Chourdakis (2005). In particular, we examine the trade-off between modelfitting performance and required calibration time under FFT and FRFT methods. 
This paper is organized as follows. Section 2 sets out the Lévy framework and provides a reformulated version of the Lévy-Kintchine formula. Section 3 shows how this reformulation can be used to derive the parameters of the VG model; this section also revisits the earlier work of Geman (2002). Section 4 sets out the risk neutral specification for the VG model which is used for pricing purposes. Section 5 outlines the FFT and FRFT approaches to option valuation, and section 6 sets out and discusses the empirical calibrations of the BS and VG model under FFT and FRFT, based on a weekly data set for 2007. Section 7 concludes.

\section{The Lévy Framework and Asset Pricing}

We begin with the definition of a Lévy process and the Lévy-Kintchine formula for infinitely divisible distributions.

Definition 2.1 A cadlag stochastic process $X=\left\{X_{t} ; t \geqslant 0\right\}$, on $(\Omega, F, P)$ with values in $\Re$ is called a Lévy process if it satisfies the following properties:

[L1] each $X_{0}=0$ a.s.

[L2] $X_{t}$ has independent and stationary increments, i.e.

(i) for every increasing sequence of times $t_{0}<t_{1}<t_{2}<\ldots<t_{n}$ the random variables $X_{t_{0}}, X_{t_{1}}-X_{t_{0}}, \ldots, X_{t_{n}}-X_{t_{n-1}}$ does not depend on $t$.

(ii) $\quad X_{t+h}-X_{t} \stackrel{D}{=} X_{t+h-t}=X_{h}$, i.e. the distribution of $X_{t+h}-X_{t}$ does not depend on $t$.

[L3] $X_{t}$ is stochastically continuous, i.e. 


$$
\lim _{h \rightarrow 0} P\left(\left|X_{t+h}-X_{t}\right|>\varepsilon\right), \forall \varepsilon>0
$$

Condition [L3] implies that for a given (deterministic) time $t$ the probability of having a jump at $t$ is zero, i.e. discontinuities (jumps) do not occur at deterministic times and so must occur at random times.

\subsection{The Lévy-Kintchine Formula}

We now use the Lévy-Kintchine formula to derive distributional characteristics of a Lévy process.

Theorem 2.1 $F \in M_{1}(\mathfrak{R})$ is infinitely divisible if there exist scalars $a, b \in \mathfrak{R}$ and $a$ measure $v$ satisfying $v(\{0\})=0$ and $\int_{\mathfrak{R} \backslash\{0\}}\left(|x|^{2} \wedge 1\right)(d x)<\infty$ such that for all $s \in \mathfrak{R}$ :

$$
\Phi_{F}(s)=\exp \left[i a s-\frac{1}{2} s^{2} b^{2}+\int_{\Re}\left[e^{i s x}-1-i s x \mathrm{I}_{\{-1,1\}}(x)\right] v(d x)\right]
$$

Conversely any mapping of the above form is the characteristic function of an infinitely divisible probability measure on $\mathfrak{R}$. Thus the parameters " $a$ ", " $b$ " and the measure " $v$ " characterize the distribution of the underlying infinitely divisible random variable and $\left(a, b^{2}, v\right)$ is known as the characteristic triplet or Lévy triplet of the underlying infinitely divisible random variable.

The derivation of equation (1) can be found in Sato (1999), Applebaum (2004) or Cont and Tankov (2004). The last term in (1) can be written as:

$$
\exp \left[\int_{|x| \leq 1}\left[e^{i s x}-1-i s x\right] v(d x)\right] \exp \left[\int_{|x|>1}\left[e^{i s x}-1\right](d x)\right]
$$


Following Applebaum (2004), we set $v(A)=\lambda F(A)$ and equation (1) becomes:

$$
\exp \left[\int_{|x| \leq 1} \lambda_{s j}\left[e^{i s x}-1-i s x\right] F(d x)\right] \exp \left[\int_{|x|>1} \lambda_{b j}\left[e^{i s x}-1\right] F(d x)\right]
$$

where "sj" and " $b j "$ stand for small jumps and big jumps respectively.

The last part of equation (3) is the characteristic function of "big" random jump sizes $|x|>1$, controlled by a compound Poisson distribution with intensity:

$$
\lambda_{b j}=\lambda \int_{|x|>1} F(d x)
$$

and the jump distribution:

$$
F_{|J|>1}(d x)=\frac{v(d x) \mathbf{I}_{|x|>1}}{\lambda_{b j}}=\frac{v(d x) \mathbf{I}_{|x|>1}}{\lambda \int_{|x|>1} F(d x)}
$$

where $\lambda=\lambda_{b j}+\lambda_{s j}$ and the random variable, $J$ describing the jumps of all sizes (with intensity of jumps of all sizes $v(\mathfrak{R})=\lambda F(\Re)=\lambda)$ has distribution $F(x)$. Thus:

$$
F_{|J|>1}(\{|x|>1\})=\frac{v(\{|x|>1\})}{\lambda \int_{|x|>1} F(d x)}=1
$$

Consequently, the last part in equation (3) is the characteristic function of the Compound Poisson process $C P\left(\lambda_{b j}, F_{|J|>1}(d x)\right)$.

For its part, the first part in equation (3) is intuitive and can be seen as a limit corresponding to $\varepsilon_{i} \rightarrow 0$ as $i \rightarrow \infty$, viz.:

$$
\exp \left[\sum_{i}\left\{\frac{\varepsilon_{i}}{s j} \int_{\varepsilon_{i}<x \mid \leq 1}\left[e^{i s x}-1-i s x\right]\right\}(d x)\right]
$$




$$
=\exp \left[\sum_{i}\left\{\lambda_{s j}^{\varepsilon_{i}} E^{F_{\varepsilon_{i}} \triangleleft|| \leq 1}\left[e^{i s J}-1\right]-i s \lambda_{s j}^{\varepsilon_{i}} E^{F_{\varepsilon_{i}} \backslash \| \mid \leq 1}|J|\right\}\right]
$$

(since $J$ is $F$-distributed)

$$
\begin{aligned}
& =\exp \left[\sum_{\mathrm{i}}\left\{\lambda_{s j}^{\varepsilon_{i}} \int\left[e^{i s x}-1-i s x\right] F^{\varepsilon_{i}<x \mid \leq 1}(d x)\right\}\right] \\
& \rightarrow \exp \left[\int_{|x| \leq 1} \lambda_{s j}\left[e^{i s x}-1-i s x\right] F(d x)\right] \text { as } i \rightarrow \infty
\end{aligned}
$$

where $\lambda_{s j}=\lambda_{s j}^{\varepsilon_{1}}+\lambda_{s j}^{\varepsilon_{2}}+\ldots .+\lambda_{s j}^{\varepsilon_{i}}+\ldots$. is the overall intensity of small jumps. The limit in equation (7) is the characteristic function of a compensated (mean subtracted) square integrable random variable (see Kyprianou (2006)). For a general $n$ each $\lambda_{s j}^{\varepsilon_{n}}$ and $F_{\varepsilon_{n}<J \mid \leq 1}(d x)$ are given respectively by:

$$
\begin{aligned}
& \lambda_{s j}^{\varepsilon_{n}}=v\left(\varepsilon_{n}<|x|<\varepsilon_{n-1}\right) \\
& F_{\varepsilon_{n}<x \mid<\varepsilon_{n-1}}\left(\frac{v(d x) 1_{\varepsilon_{n}<x \mid<\varepsilon_{n-1}}}{\lambda_{s j}^{\varepsilon_{n}}}\right)
\end{aligned}
$$

The overall intensity of small jumps with magnitude less than one is then:

$$
\begin{aligned}
\lambda_{s j} & =v\left(\left\{\varepsilon_{1}<|x| \leq 1\right\} \cup\left\{\varepsilon_{2}<|x| \leq 1\right\} \cup \ldots \cup\left\{\varepsilon_{n}<|x| \leq 1\right\} \cup \ldots\right) \\
& =v(|x| \leq 1) \\
& =\lambda \int_{|x| \leq 1} F(d x)
\end{aligned}
$$

Now consider an arbitrary summand in equation (6):

$$
\begin{aligned}
& \exp \left\{\lambda_{s j}^{\varepsilon_{i}} E^{F_{\varepsilon_{i}<J \mid \leq 1}}\left[e^{i s J}-1\right]-i s \lambda_{s j}^{\varepsilon_{i}} E^{F_{\varepsilon_{i}<J \mid J 1}}|J|\right\} \\
= & \exp \left[\lambda_{s j}^{\varepsilon_{i}} E^{F_{\varepsilon_{i}<J \mid \leq 1}}\left[e^{i s J}-1\right]\right] \exp \left[-i s \lambda_{s j}^{\varepsilon_{i}} E^{F_{\varepsilon_{i}<J \mid \leq 1}}|J|\right]
\end{aligned}
$$


The first part in (11) corresponds to the characteristic function of a random variable $\sum_{j=1}^{N} J_{j} \mathrm{I}_{\varepsilon_{i}<J J_{j} \mid \leq 1} \sim C P\left(\lambda_{s j}^{\varepsilon_{i}}, F_{\varepsilon_{i}<J \mid \leq 1}(d x)\right)$ with $\lambda_{s j}^{\varepsilon_{n}}$ and $F_{\varepsilon_{i}<J \mid \leq 1}$ given by equation (8) and equation (9) respectively (see Cont and Tankov (2000))). Here $N \sim \operatorname{Poisson}\left(\lambda_{s j}^{\varepsilon_{i}}\right) \quad$ and hence we obtain $E\left[\sum_{j=1}^{N} J_{j} \mathrm{I}_{\varepsilon_{i} \backslash J_{j} \mid \leq 1}\left|=E[N] E^{F_{\varepsilon_{i} \backslash\langle| j \leq 1}}\right| J\left|=\lambda_{s j}^{\varepsilon_{i}} E^{F_{\varepsilon_{i}}<J \mid \leq 1}\right| J \mid\right.$, implying that the second part in equation (11) is the characteristic function of a constant equal to the mean of $C P\left(\lambda_{s j}^{\varepsilon_{i}}, F_{\varepsilon_{i}<J \mid \leq 1}(d x)\right)$. Hence equation (11) is the characteristic function of a compensated (mean subtracted) compound Poisson random variable of small jumps $(s j)$, which we denote as $\sum_{j=1}^{N} J_{j} \mathrm{I}_{\varepsilon_{i}<J_{j} \mid \leq 1}-E^{F_{\varepsilon_{i}}<J \mid \leq 1}|J| \sim C P^{c}\left(\lambda_{s j}^{\varepsilon_{i}}, F_{\varepsilon_{i}<J_{j} \mid \leq 1}(d x)\right)$. Since this is true for each summand, equation (6) is the characteristic function of the sum of a possibly infinite number of compensated compound Poisson random variables:

$$
C P^{c}\left(\lambda_{s j}^{\varepsilon_{1}}, F_{\varepsilon_{1}<J \mid \leq 1}(d x)\right)+C P^{c}\left(\lambda_{s j}^{\varepsilon_{2}}, F_{\varepsilon_{2}<J \mid \leq 1}(d x)\right)+\ldots+C P^{c}\left(\lambda_{s j}^{\varepsilon_{i}}, F_{\varepsilon_{i}<J \mid \leq 1}(d x)\right)+\ldots
$$

The compensation is required to obtain the convergence, as in equation (7), of numerous small jumps described by possibly infinite number of compensated compound Poisson random variables, to a compensated square integrable random variable whose characteristic function is exactly the first part of equation (3).

Thus equation (1) characterizes the limiting distribution of the sum of $a+N\left(0, b^{2}\right)+C P\left(\lambda_{b j}, F_{|J|>1}(d x)\right)+\sum_{i}\left\{C P^{c}\left(\lambda_{s j}^{\varepsilon_{1}}, F_{\varepsilon_{1}<|J| \leq 1}(d x)\right)\right\}$ where the rates and distributions of big and small jumps are as described above.

\subsection{The Lévy-Kintchine Formula in Asset Pricing}


We now replace the noise process in the standard Black-Scholes dynamics with an arbitrary Lévy process $X_{t}$ :

$$
\frac{d S_{t}}{S_{t}}=\hat{\mu} d t+d X_{t}
$$

where the usual volatility $\sigma$ is set to 1 for simplicity. Integrating on $\left[t_{1}, t_{2}\right]$, we obtain an infinitely divisible random variable describing the random evolution of log returns on $\left[t_{1}, t_{2}\right]:$

$$
\begin{gathered}
\ln \left(\frac{S_{t_{2}}}{S_{t_{1}}}\right)=\hat{\mu}\left(t_{2}-t_{1}\right)+X_{t_{2}}-X_{t_{1}} \\
\quad \stackrel{D}{=} \hat{\mu}\left(t_{2}-t_{1}\right)+X_{\left(t_{2}-t_{1}\right)}
\end{gathered}
$$

by [L2](ii) in Definition 2.1. According to the Lévy-Kintchine formula the distribution of $X_{t_{2}}-X_{t_{1}}$ is characterized by the characteristic function of an infinitely divisible random variable given by:

$$
E\left[e^{i s X_{\left(12-t_{1}\right)}}\right]=\exp \left\{\left(t_{2}-t_{1}\right)\left[i a s-\frac{1}{2} s^{2} b^{2}\right]+\int_{\mathfrak{R}\{\{0\}}\left[e^{i s x}-1-i s x \mathrm{I}_{\{-1,1\}}(x)\right] v(d x)\right\}
$$

Thus following section 2.1, we can rewrite (14) as:

$$
\begin{array}{r}
\ln \left(\frac{S_{t_{2}}}{S_{t_{1}}}\right)^{D}=\hat{\mu}\left(t_{2}-t_{1}\right)+a\left(t_{2}-t_{1}\right)+\mathrm{N}\left(0,\left[\sqrt{t_{2}-t_{1}}\right] b\right)^{2}+C P\left[\left(t_{2}-t_{1}\right) \lambda_{b j}, F_{|| \mid>1}(d x)\right] \\
+\lim _{\substack{i \rightarrow \infty \\
\varepsilon_{i} \rightarrow 0}} \sum_{i}\left\{C P ^ { c } \left[\left(t_{2}-t_{1}\right) \lambda_{s j}^{\varepsilon_{j}}, F_{\varepsilon_{i}}|| \mid \leq 1 \leq 1\right.\right. \\
\end{array}
$$


where $\mu=\hat{\mu}+a$. The rates $\lambda_{b j}$ and $\lambda_{s j}^{\varepsilon_{i}}$ as well as the distributions $F_{|J|>1}(d x)$ and $F_{\varepsilon_{i}<|J| \leq 1}(d x)$ of big and small jumps, respectively, are as described in section 2.1 .

\subsection{Changing Time through Subordinator Processes}

Subordinators are stochastic processes which are used to introduce randomness in the time axis. An intuitive interpretation of the purpose of such stochastic processes, replacing calendar spacing of time with 'business' spacing of time, is discussed in Geman (2002). The occurrence of events is then modeled using business time instead of calendar time.

Definition 2.2 (Subordinator) Let $\left\{X_{t} ; t \geqslant 0\right\}$ be a Lévy process such that $X_{t}$ has the Lévy triplet $\left(a, b^{2}, v\right)$. Then $X_{t}$ is an increasing process in $t$ if and only if $v(-\infty, 0]=0, b^{2}=0, \int_{0}^{1} x v(d x)<\infty$ and $d=a-\int_{0}^{1} x v(d x)>0$. Such an increasing process is known as a subordinator.

In this case $X_{t}$ can be expressed as the sum of both its linear drift and its jumps over times 0 to $t$ :

$$
X_{t}=d t+\int_{[0, t] \times \mathcal{R}} x J_{X}(d s \times d x)=d t+\sum_{\substack{0 \leq s \leq t \\\left|\Delta X_{s}\right| \geq 1}} \Delta X_{s}, t \geq 0
$$

and its characteristic function is expressed as:

$$
E\left[e^{i s X_{t}}\right]=\exp \left[t\left\{i d s+\int\left(e^{i s x}-1\right) v(d x)\right\}\right.
$$

where $d=a-\int_{|x| \leq 1} x v(d x)$. 
Thus in the case of the subordinator drift being positive and there being no negative jumps in the time process, the diffusion component needs to be zero, since otherwise there will be a positive probability of a negative change in time over any interval. Consequently, positive jumps are the only source of randomness and finite variation ensures that small jumps are summable. This implies that the usual compensation of small jumps in the Lévy-Kintchine formula (to ensure the integrability of the Lévy measure) is not needed and the compensation part can be adjusted with the drift of the process to give a new drift. (Further details can be found in Cont and Tankov (2004) and Sato (199)).)

The following theorem shows that when a Lévy process modeling return dynamics is subordinated by a subordinator (modeling time change) then the resultant process is still a Lévy process. Moreover it shows how to obtain the characteristics of the resultant process.

Theorem 2.3 Let $T_{t}$ be a subordinator with Lévy measure $\tilde{v}$, drift $d$. Its distribution at time $t, P_{T_{t}}$ is characterized by the equation (18) and let $\lambda=P_{T_{1}}$. Further assume that $X_{t}$ is a $\mathfrak{R}$-valued Lévy process with Lévy triplet $\left(a, b^{2}, v\right)$. Its distribution $P_{X_{t}}$, at $t>0$ is characterized by equation (1) and let $\mu=P_{X_{1}}$. Then provided the processes $X_{t}$ and $T_{t}$ are independent, the process defined as

$$
Y_{t}(\omega)=X_{T_{t(\omega)}}(\omega) \quad t \geq 0
$$

is also a Lévy process. The distribution of $Y_{t}$ is given by:

$$
P\left[Y_{t} \in B\right]=\int_{0}^{\infty} \mu^{s}(B) \lambda^{t}(d s), B \in \aleph(\Re)
$$


The Lévy triplet $\left(a_{y}, b_{y}^{2}, v_{y}\right)$ of $Y_{t}$ is given by:

$$
\begin{aligned}
a_{y} & =d \cdot a+\int_{0}^{\infty} \tilde{v}(d s) \int_{|x| \leq 1} x \mu^{s}(d x) \\
b_{y}^{2} & =d \cdot b^{2} \\
v_{y}(B) & =d \cdot v(B)+\int_{0}^{\infty} \mu^{s}(B) \tilde{v}(d s), \quad B \in \aleph(\mathfrak{R} \backslash\{0\})
\end{aligned}
$$

The proof of the above theorem can be found in Sato (1999). We now explore VG process using theorem 2.3 to relate its dynamics to equation (16).

\section{Revisiting Variance-Gamma as a Time-Changed Process}

The Variance Gamma (VG) process introduced by Madan et al. (1998) involves timechanging Brownian motion by an increasing gamma process. Gamma random variables are infinitely divisible and so, according to the Lévy-Kintchine formula, a gamma process can be obtained such that:

$$
T_{t+s}-T_{t}=\Gamma\left(\frac{s}{\gamma}, \frac{1}{\gamma}\right) \text { in general } T_{t}=\Gamma\left(\frac{t}{\gamma}, \frac{1}{\gamma}\right)
$$

where $\Gamma(\alpha, \beta)$ has the density:

$$
f(x)=\frac{\beta^{\alpha}}{\Gamma(\alpha)} x^{\alpha-1} e^{\beta x} ; x>0, \alpha>0, \beta>0
$$

(see Cont and Tankov, [2004]).

We now note the following two lemmas:

Lemma 3.1 The generating triplet for the $\Gamma(\alpha, \beta)$ distribution is $\left(0,0, v^{s}\right)$, where the Lévy measure $v^{s}$ is given by:

$$
v^{s}(d x)=\frac{\alpha}{x} e^{-\beta x} d x, x>0
$$


It then follows that the gamma process $T_{t}$ in equation (24) with $\alpha=1 / \gamma$ and $\beta=1 / \gamma$ is a subordinator with triplet $\left(0,0, t v^{s}\right)$.

Lemma 3.2 If $K_{p}$ is a modified Bessel function of third kind, then:

$$
\begin{aligned}
K_{p}(x) & =\frac{1}{2}\left(\frac{x}{2}\right)^{p} \int_{0}^{\infty} e^{-t-\frac{x^{2}}{4 t}} t^{-p-1} d t ; x>0, p \in \mathfrak{R} \\
K_{n+\frac{1}{2}}(x) & =\sqrt{\left(\frac{\pi}{2}\right)} x^{-\frac{1}{2}} e^{-x}\left(1+\sum_{i=1}^{n} \frac{(n+i) !}{(n-i) ! i !}(2 x)^{-i}\right)
\end{aligned}
$$

The derivation of the above Lemma can be found in Watson [1944].

Now consider the process $X_{t}=\sigma B_{t}+\theta t$, where $B_{t}$ is a standard Brownian motion with volatility and drift parameters $\sigma>0$ and $\theta \in \mathfrak{R}>0$. The VG process is then defined as the process $Y$ subordinated to $X$ by the $\Gamma$-subordinator $T$ :

$$
Y_{t}:=X_{T_{t}}=\sigma B_{t}+\theta T_{t}
$$

The gamma process is characterized as in equation (24) so that it ensures the mean rate $t$ and variance $\gamma t$ with the probability density:

$$
f_{T_{t}}(x)=\frac{\left(\frac{1}{\gamma}\right)^{\frac{t}{\gamma}}}{\Gamma\left(\frac{t}{\gamma}\right)} x^{\frac{t}{\gamma}-1} e^{-\frac{x}{\gamma}}
$$

With this parameterization the Laplace transform of the gamma subordinator is:

$$
E\left[e^{-s T_{t}}\right]=(1+s \gamma)^{-\frac{t}{\gamma}}
$$

Equation (29) shows that, conditional on a jump of size $T_{t}=s$ in the time change, the change of the process $Y_{t}$ is normally distributed with mean $\theta s$ and variance $\sigma^{2} s$. The 
characteristic function of the $\mathrm{VG}$ process is then obtained by conditioning on the subordinator, viz.:

(using equation (31))

$$
\begin{aligned}
\Phi_{Y_{t}} & =E\left[e^{i s\left(\sigma B_{T_{t}}+\theta T_{t}\right)}\right] \\
& =\int_{0}^{\infty} E\left[e^{i s\left(\sigma B_{T_{t}}+\theta T_{t}\right)} \mid T_{t}=u\right] f_{T_{t}}(u) d u \\
& =\int_{0}^{\infty} e^{i s \theta u-\frac{1}{2} s^{2} \sigma^{2} u\left(\sigma B_{T_{t}}+\theta T_{t}\right)} f_{T_{t}}(u) d u \\
& =\left(1+\left[\frac{1}{2} s^{2} \sigma^{2}-i s \theta\right] \gamma\right)^{\frac{t}{\gamma}}
\end{aligned}
$$

$$
=\left(\frac{1}{1-i s \theta+\frac{1}{2} s^{2} \sigma^{2}}\right)^{\frac{t}{\gamma}}
$$

Applying Theorem 2.3 then yields the Lévy triplet of the VG process. From equation (21):

$$
\begin{aligned}
a_{v g} & =[\text { drift subordinator }][\text { drift subordinate }]+\int_{0}^{\infty} \tilde{v}(d x) \cdot \int_{-1}^{1} y P_{\sigma B_{1}+\theta}^{s}(d y) \\
& =0 .[\text { drift subordinate }]+\int_{0}^{\infty} \frac{\left(\frac{1}{\gamma}\right)}{x} e^{-\frac{x}{\gamma}} d x \cdot \int_{-1}^{1} y P_{\sigma B_{1}+\theta}^{s}(d y)
\end{aligned}
$$

(using Lemma 3.1)

$$
=\int_{0}^{\infty} \frac{\left(\frac{1}{\gamma}\right)}{x} e^{-\frac{x}{\gamma}} d x \cdot \int_{-1}^{1} y \frac{1}{\sigma \sqrt{2 \pi s}} e^{-\frac{(y-\theta s)^{2}}{2 \sigma^{2} s}} d y \in \mathfrak{R}
$$

From equation (22):

$$
b_{v g}=[\text { drift subordinator }][\text { drift subordinate }]=0
$$

also using Lemma 3.1.

From equation (23):

$$
\begin{aligned}
v_{v g} & =[\text { drift subordinator }] . \text { [drift subordinate }]+\int_{0}^{\infty} P_{\sigma B_{1}+\theta}^{s}(d x) \tilde{v}(d s) \\
& =0+\int_{0}^{\infty} \frac{1}{\sigma \sqrt{2 \pi s}} e^{-\frac{(x-\theta s)^{2}}{2 \sigma^{2} s}} \frac{\alpha}{s} e^{-\beta s} d s
\end{aligned}
$$

[with $\alpha=1 / \gamma$ and $\beta=1 / \gamma$ using Lemma 3.1]

$$
=\frac{\alpha}{\sigma \sqrt{2 \pi}} d x \int_{0}^{\infty} \frac{1}{\sigma \sqrt{2 \pi s}} e^{-\frac{(x-\theta s)^{2}}{2 \sigma^{2} s}} s^{-\frac{3}{2}} e^{-\beta s} d s
$$


Since $e^{-\frac{(x-\theta)^{2}}{2 \sigma^{2} s}} e^{-\beta s}=e^{\frac{x \theta}{\sigma^{2}}} e^{\left[-\left(\beta+\frac{\theta^{2}}{2 \sigma^{2}}\right) s-\left(\frac{x^{2}}{2 \sigma^{2}}\right) \frac{1}{s}\right]}$ we now use (35) to obtain:

$$
\begin{aligned}
v_{v g} & =\frac{\alpha}{\sigma \sqrt{2 \pi}} d x \int_{0}^{\infty} e^{-\frac{\left(x-\theta \theta^{2}\right)^{2}}{2 \sigma^{2} s}} s^{-\frac{3}{2}} e^{-\beta s} d s \\
& =\frac{\alpha}{\sigma \sqrt{2 \pi}} e^{\frac{x \theta}{\sigma^{2}}} d x \int_{0}^{\infty} s^{-\frac{3}{2}} \exp \left[-\left(\beta+\frac{\theta^{2}}{2 \sigma^{2}}\right) s-\left(\frac{x^{2}}{2 \sigma^{2}}\right) \frac{1}{s}\right] d s
\end{aligned}
$$

To evaluate the integral in equation (36) we need to use equation (27). We then set $\kappa=\left(\beta+\frac{\theta}{2 \sigma^{2}}\right)$ and $s^{\prime}=\kappa s$ and rearrange the integrand in equation (36) to obtain:

$$
\begin{aligned}
& \int_{0}^{\infty}\left(\frac{s^{\prime}}{\kappa}\right)^{-\frac{3}{2}} \exp \left[-s^{\prime}-\left(\frac{\frac{2 x^{2} \kappa}{\sigma^{2}}}{4 s^{\prime}}\right)\right] \frac{1}{\kappa} d s^{\prime} \\
& =\int_{0}^{\infty} s^{\prime-\frac{3}{2}} \exp \left[-s^{\prime}-\left(\frac{\left(\sqrt{\frac{2 x^{2} \kappa}{\sigma^{2}}}\right)^{2}}{4 s^{\prime}}\right)\right] d s^{\prime} \\
& =\sqrt{\kappa} K_{\frac{1}{2}}\left(\sqrt{\frac{2 x^{2} \kappa}{\sigma^{2}}}\right)\left[\frac{1}{2}\left(\sqrt{\frac{\frac{2 x^{2} \kappa}{\sigma^{2}}}{2}}\right)^{\frac{1}{2}}\right]^{-1}
\end{aligned}
$$

Consequently (36) becomes: 


$$
\begin{aligned}
v_{v g} & =\frac{\alpha}{\sigma \sqrt{2 \pi}} e^{\frac{x \theta}{\sigma^{2}}} \frac{\sqrt{\kappa} K_{\frac{1}{2}}\left(\sqrt{\frac{2 x^{2} \kappa}{\sigma^{2}}}\right)}{\left.\left[\frac{\sqrt{\frac{2 x^{2} \kappa}{\sigma^{2}}}}{2}\right)^{\frac{1}{2}}\right]} d x \\
= & \frac{\alpha}{\sigma \sqrt{2 \pi}} e^{\frac{x \theta}{\sigma^{2}}} \frac{\sqrt{\kappa} \sqrt{\frac{\pi}{2}} \cdot \frac{1}{\left(\sqrt{\frac{2 x^{2} \kappa}{\sigma^{2}}}\right)^{\frac{1}{2}}}}{\left[\frac{\left.\sqrt{\frac{2 x^{2} \kappa}{\sigma^{2}}}\right)^{\frac{1}{2}}}{2}\right)^{2}} d x \\
= & \frac{\alpha}{|x|} \exp \left(\frac{x \theta}{\sigma^{2}}-\frac{|x|}{\sigma} \sqrt{2 \kappa}\right) d x \\
= & \frac{1}{\gamma|x|} \exp \left(\frac{x \theta}{\sigma^{2}}-\frac{|x|}{\sigma} \sqrt{\frac{2}{\gamma}+\frac{\theta^{2}}{\sigma^{2}}}\right) d x
\end{aligned}
$$

The last equality follows by plugging back the value of $\kappa$ with $\beta=1 / \gamma$.

The drifted Brownian motion, without time change, describes the assets log return through two parameters $\mu$ and $b$ as in equation (16) (without compound Poisson parts). As equation (29) shows, the VG is a Brownian motion with a change of calendar time to business time made by a gamma process. The parameters $a_{v g}$ and $b_{v g}$ play the same role for the VG process as parameters $a$ and $b$ in equation (16) for a general Lévy process. However, $b_{v g}$ being zero, equation (16) shows that the dynamics of the log returns have no diffusion but only jumps. Furthermore $v_{v g}$ completely describes the rate and distribution of both small and big jumps, as explained in section 2.1.

\subsection{Geman's misspecification}


The derivation of the Lévy measure in the previous section helps us recognize that with the specification of the parameters in Geman (2002), the form of the Lévy measure mentioned therein is not correct (although her numerical work was not affected by this error). We now derive the expression of Lévy measure as used by Geman and report the correct specification of the parameters.

Geman et al. (2001) show that the VG process may be expressed as the difference of two independent gamma processes:

$$
Y_{t}=G_{t}^{p}-G_{t}^{n}
$$

where $G_{t}^{p}$ and $G_{t}^{n}$ are interpreted as the price changes from positive and negative shocks respectively. Given equation (39), equations (31) and (32) then suffice to demonstrate that:

$$
\begin{aligned}
\frac{1}{1-i s \theta \gamma+\frac{1}{2} s^{2} \sigma^{2} \gamma} & =\left(\frac{1}{1-i s \eta_{p}}\right) \cdot\left(\frac{1}{1-i s \eta_{n}}\right) \\
& =\frac{1}{1-i s\left(\eta_{p}-\eta_{n}\right)+s^{2} \eta_{p} \eta_{n}}
\end{aligned}
$$

which is equivalent to:

$$
\begin{gathered}
\eta_{p}-\eta_{n}=\theta \gamma \\
\eta_{p} \eta_{n}=\frac{\sigma^{2} \gamma}{2}
\end{gathered}
$$

Geman [2002] specified the solutions of (41) and (42) as:

$$
\begin{aligned}
& \eta_{p}=\frac{1}{\sqrt{\frac{\theta^{2} \gamma^{2}}{4}+\frac{\theta^{2} \gamma}{2}}-\frac{\theta \gamma}{2}} \\
& \eta_{n}=\frac{1}{\sqrt{\frac{\theta^{2} \gamma^{2}}{4}+\frac{\theta^{2} \gamma}{2}}+\frac{\theta \gamma}{2}}
\end{aligned}
$$


She went on to argue that with these specifications the Lévy measure of the VG process can be written as:

$$
v_{v g}(d x)=\left\{\begin{array}{l}
C \frac{e^{-M x}}{x} d x \text { if } x>0 \\
C \frac{e^{-G x}}{x} d x \text { if } x<0
\end{array}\right.
$$

where $C=\frac{1}{\gamma}, G=\frac{1}{\eta_{n}}, M=\frac{1}{\eta_{p}}$.

However using the derived form of Lévy measure (see equation (38)), one can see that solutions (43) and (44) do not yield the expression of the Lévy measure (45). Moreover (43) and (44) do not even satisfy (41) and (42).

To show the correct approach, we now solve equation (41) and (42) separately for $\eta_{p}$ and $\eta_{n}$. For $\eta_{p}$ we write equation (41) as $\eta_{p}=\eta_{n}+\theta \gamma$. Then from equation (42) we obtain:

$$
\begin{aligned}
\left(\eta_{n}+\theta \gamma\right) \eta_{n} & =\frac{\sigma^{2} \gamma}{2} \\
\Rightarrow \eta_{n} & =\frac{-2 \theta \eta \pm \sqrt{4 \theta^{2} \gamma^{2}+8 \sigma^{2} \gamma}}{4} \\
& \stackrel{(+)}{\frac{\theta^{2} \gamma^{2}}{4}+\frac{\sigma^{2} \gamma}{2}}-\frac{\theta \gamma}{2}
\end{aligned}
$$

Then again from equation (42) we obtain:

$$
\eta_{p}=\frac{1}{\frac{2}{\sigma^{2} \gamma}\left(\sqrt{\frac{\theta^{2} \gamma^{2}}{4}+\frac{\sigma^{2} \gamma}{2}}-\frac{\theta \gamma}{2}\right)}
$$

For $\eta_{n}$ we write equation (41) as $\eta_{n}=\eta_{p}-\theta \gamma$. Equation (42) now implies: 


$$
\begin{aligned}
\left(\eta_{p}-\theta \gamma\right) \eta_{p} & =\frac{\sigma^{2} \gamma}{2} \\
\Rightarrow \eta_{p} & =\frac{-2 \theta \eta \pm \sqrt{4 \theta^{2} \gamma^{2}+8 \sigma^{2} \gamma}}{4} \\
& \stackrel{(+)}{=} \sqrt{\frac{\theta^{2} \gamma^{2}}{4}+\frac{\sigma^{2} \gamma}{2}}+\frac{\theta \gamma}{2}
\end{aligned}
$$

Then once again from (42) we obtain:

$$
\eta_{n}=\frac{1}{\frac{2}{\sigma^{2} \gamma}\left(\sqrt{\frac{\theta^{2} \gamma^{2}}{4}+\frac{\sigma^{2} \gamma}{2}}+\frac{\theta \gamma}{2}\right)}
$$

Our solutions satisfy equation (40). Moreover using equation (38), we now prove that our solutions produce the form of Lévy measure mentioned by Geman.

From equation (38) for $x>0$ we obtain:

$$
\begin{aligned}
v_{v g}(d x) & =\frac{1}{\gamma|x|} \exp \left[x\left(\frac{\theta}{\sigma^{2}}-\frac{1}{\sigma} \sqrt{\frac{2}{\gamma}+\frac{\theta^{2}}{\sigma^{2}}}\right)\right] d x \\
& =\frac{1}{\gamma|x|} \exp \left[-x \frac{2}{\sigma^{2} \gamma}\left(\sqrt{\frac{\theta^{2} \gamma^{2}}{4}+\frac{\sigma^{2} \gamma}{2}}-\frac{\theta \gamma}{2}\right)\right] d x \\
& =C \frac{e^{-M x}}{x}, x>0
\end{aligned}
$$

where $C=\frac{1}{\gamma}, M=\frac{1}{\eta_{p}}$ with $\eta_{p}$ given by equation (46).

Similarly from equation (38), since for $x<0 ;|x|=-x$ i.e. $\mathrm{x}=-|x|$, we obtain:

$$
\begin{aligned}
v_{v g}(d x) & =\frac{1}{\gamma|x|} \exp \left[-\mid x\left(\frac{\theta}{\sigma^{2}}+\frac{1}{\sigma} \sqrt{\frac{2}{\gamma}+\frac{\theta^{2}}{\sigma^{2}}}\right)\right] d x \\
& =\frac{1}{\gamma|x|} \exp \left[-|x| \frac{2}{\sigma^{2} \gamma}\left(\sqrt{\frac{\theta^{2} \gamma^{2}}{4}+\frac{\sigma^{2} \gamma}{2}}+\frac{\theta \gamma}{2}\right)\right] d x \\
& =C \frac{e^{-G|x|}}{x}, x<0
\end{aligned}
$$


where $C=\frac{1}{\gamma}, G=\frac{1}{\eta_{n}}$ with $\eta_{n}$ given by equation (47). Equation (48) and (49) together imply (45). QED.

\section{Risk-Neutral Specifications}

To price the option we use the risk-neutral characteristic function. To obtain the latter, we start with the characteristic function of the VG model under the real measure, given by equation (32), rewritten as:

$$
\Phi_{Y_{t}}(s)=\exp \left\{-\frac{t}{\gamma} \ln \left(1-i s \theta \gamma+\frac{1}{2} s^{2} \sigma^{2} \gamma\right)\right\}
$$

We can extract two parts from equation (50): the drift part $\mu=0$ and the non-drift part $\phi(s)=-\frac{t}{\gamma} \ln \left(1-i s \theta \gamma+\frac{1}{2} s^{2} \sigma^{2} \gamma\right)$. The drift part under the risk-neutral measure can now be obtained (see Shiryaev, [1999]) as:

$$
\mu^{r n}(s)=i\left[r-\frac{\phi(-i)}{t}\right] s t=i\left[r+\frac{1}{\gamma} \ln \left(1-\theta \gamma-\frac{1}{2} \sigma^{2} \gamma\right)\right] s t
$$

The risk-neutral characteristic function can then be obtained as:

$$
\begin{aligned}
\Phi_{Y_{t}}^{r n}(s) & =\exp \left\{\mu^{r n}(s)+\phi(s)\right\} \\
& =\exp \left\{i\left[r+\frac{1}{\gamma} \ln \left(1-\theta \gamma-\frac{1}{2} \sigma^{2} \gamma\right)\right] s t-\frac{t}{\gamma} \ln \left(1-i s \theta \gamma+\frac{1}{2} s^{2} \sigma^{2} \gamma\right)\right\}
\end{aligned}
$$

Similarly, the real measure characteristic function of the Black-Scholes Brownian motion $B_{t} \sim N(\bar{\mu} t, \sigma t)$ is:

$$
\Phi_{B_{t}}(s)=\exp \left\{i s \bar{\mu} t-\frac{1}{2} s^{2} \sigma^{2} t\right\}
$$


and its risk-neutral equivalent is

$$
\Phi_{B_{t}}^{r n}(s)=\exp \left\{i\left(r-\frac{1}{2} \sigma^{2}\right) s t-\frac{1}{2} s^{2} \sigma^{2} t\right\}
$$

Our empirical study is conducted under the risk-neutral measure utilizing the risk-neutral characteristic functions (52) and (54).

\section{Pricing with FFT and FRFT}

Now consider $s_{t}=\ln \left(S_{t}\right)$ and $k=\ln (K)$ where $K$ is the strike price of the option. As in Carr and Madan (1999), the value of a European call with maturity $T$ is expressed as a function of $k$ :

$$
C_{T}(k)=\int_{k}^{\infty} e^{-r T}\left(e^{s}-e^{k}\right) q_{T}(s) d s
$$

where $q_{T}(s)$ is the risk-neutral density of the $\log$ of the prices. To overcome the nonsquare integrability of $C_{T}(k)$, Carr and Madan (1999) introduced modified call prices:

$$
c_{T}(k)=e^{\alpha k} C_{T}(k), \alpha>0
$$

where $\alpha$ is known as the dampening factor. Following Carr and Madan (1999) an analytic expression for the pricing formula (55) can be obtained as:

$$
C_{T}(k)=\frac{e^{-\alpha k}}{\pi} \int_{0}^{\infty} e^{-i u k} \psi_{T}(u) d u
$$

where $\psi_{T}$ has the following analytic expression: 


$$
\psi_{T}(u)=\frac{e^{-r T} \Phi_{T}(u-(\alpha+1) i)}{\alpha^{2}+\alpha-u^{2}-i(2 \alpha+1) u}
$$

and where $\Phi$ is the characteristic function of the model for which prices are computed. Using a suitable numerical integration technique, e.g. the trapezoidal rule, the integral appearing in equation (57) can be approximated as:

$$
\int_{0}^{\infty} e^{-i u k} \psi_{T}(u) d u \approx \sum_{j=0}^{N-1} e^{-i u_{j} k} \tilde{\psi}_{T}\left(u_{j}\right) \eta
$$

where $\tilde{\psi}_{T}$ is exactly as $\psi_{T}$ with weights attached by the integration rule, and $\eta$ is grid spacing such that $u_{j}=\eta j$ where the upper limit of integration is $N$.

For some integrable function $f$, the spirit of FFT lies in approximating the continuous Fourier Transform by its discrete version:

$$
\int_{0}^{\infty} e^{-i u k} f(u) d u \approx \sum_{j=0}^{N-1} e^{-i \frac{2 \pi}{N} k j} f\left(u_{j}\right)
$$

The usual approach in the literature is to fine-tune equation (59) and (60) and then obtain the option prices through equation (57). The usual technique is to consider only the useful log-strikes near log-spots:

$$
k_{\Delta}=-\frac{N \lambda}{2}+\lambda \Delta+\ln \left(S_{0}\right) \quad \Delta=0, \ldots, N-1
$$

where $\lambda$ here is the grid length of equidistant log-strikes. For Lévy models assuming $S_{0}=1$ and then assuming $b=\frac{N \lambda}{2}$ equation (61) ensures that log-strikes range between $-b$ to $b$. We can then write the sum in equation (59) as: 


$$
\sum_{j=0}^{N-1} e^{-i u_{j} k_{\Delta}} \tilde{\psi}_{T}\left(u_{j}\right) \eta=\sum_{j=0}^{N-1} e^{-i u_{j}\left(-\frac{N \lambda}{2}+\lambda \Delta\right)} \widetilde{\psi}_{T}\left(u_{j}\right) \eta \stackrel{u_{j}=\eta j j}{=} \sum_{j=0}^{N-1} e^{-i \eta j} e^{i \eta j \frac{N \lambda}{2}} \widetilde{\psi}_{T}\left(u_{j}\right) \eta
$$

With the following notation we obtain equation (62) in the form of equation (60) which is suitable for applying FFT on the vector $f$ with components $f\left(u_{i}\right)$ :

$$
\begin{gathered}
f\left(u_{j}\right)=e^{i \eta j \frac{N \lambda}{2}} \tilde{\psi}_{T}\left(u_{j}\right) \eta \\
\eta \lambda=\frac{2 \pi}{N}
\end{gathered}
$$

Out of the three parameters $\eta, \lambda$ and $N$, two can be chosen arbitrarily and the other should satisfy equation (64), the so called FFT condition. For decent accuracy both $\eta$ and $\lambda$ have to be small; equation (64) then forces $N$ to be large. This implies a trade-off between accuracy and the number of strikes (and hence computational time).

FRFT was first introduced by Bailey and Swartztrauber (1991) and was recently incorporated into option pricing by Chourdakis (2005). It was developed to avoid the restrictive condition (64) and so provide greater flexibility in the choice of all three parameters: it allows us to choose smaller $N$, so substantially reducing substantial computational time requirements; it also allows us to calibrate the grid spacing parameters $\eta$ and $\lambda$ for greater accuracy. In effect, FRFT is a fast and easy way to compute sums of the form:

$$
\sum_{j=0}^{N-1} e^{-i 2 \pi k j \varepsilon} f_{j}
$$


for a suitable value of the fractional parameter $\varepsilon$, where the special case $\varepsilon=\frac{1}{N}$ yields the usual FFT. Once one chooses the values of $N$, of upper integration limit $a$ and of the logstrike bound $b$, the grid spacing and fractional parameters can then be obtained as:

$$
\begin{aligned}
& \eta=\frac{a}{N} \\
& \lambda=\frac{2 b}{N} \\
& \varepsilon=\frac{1}{N}=\frac{\eta \lambda}{2 \pi}
\end{aligned}
$$

In our empirical study we use $a=64, b=0.3$ and $N=32$. (A consistent way of choosing FRFT parameters and related issues are discussed in Lee [2004].) To compute $N$-point FRFT for a vector $x$, Bailey and Swartztrauber (1991) suggest defining 2N-point vectors as:

$$
\begin{aligned}
& y_{j}= \begin{cases}x_{j} e^{-i \pi j^{2} \varepsilon} & 0 \leq j<m \\
0 & m \leq j<2 m\end{cases} \\
& z_{j}= \begin{cases}e^{i \pi j^{2} \varepsilon} & 0 \leq j<m \\
e^{i \pi(j-2 m)^{2} \varepsilon} & m \leq j<2 m\end{cases}
\end{aligned}
$$

where $\varepsilon$ is as given by equation (68). The FRFT is then computed as:

$$
G_{k}(x, \varepsilon)=e^{-i \pi k^{2} \varepsilon} \otimes D_{k}^{-1}\left[D_{j}(y) \otimes D_{j}(z)\right]
$$

where $\otimes$ stands for element wise multiplication, $D_{j}($.$) is the discrete Fourier transform$ computed with the usual FFT procedure as in equation (60) and $D^{-1}$ is the inverse Fourier transform. 
Our closed form Black-Scholes prices, used in comparison, are calculated under the riskneutral measure using the following celebrated result due to Black and Scholes (1973):

$$
\Phi_{B_{t}}^{r n}(s)=\exp \left\{i\left(r-\frac{1}{2} \sigma^{2}\right) s t-\frac{1}{2} s^{2} \sigma^{2} t\right\}
$$

\section{Empirical Analysis}

We calibrate the models separately assuming FRFT and FFT as different models and do so on weekly S\&P500 traded options data over the sample period January 2007 to November 2007. For out-of-sample assessment, we consider market prices of options traded on the last week of December 2007. For calibrating the models with different specifications we minimize the RMSE defined as:

$$
R M S E=\sqrt{\frac{1}{n} \sum_{i=1}^{n}\left(C_{i}^{\text {market }}-C_{i}^{\text {model }}\right)^{2}}
$$

[TABLE 1 APPROXIMATELY HERE]

Table 1 reports the calibration results. We see that the FRFT reduces calculation time by 97-98\% relative to the FFT. This result is consistent with existing literature. We also note that this increase in speed comes at a small cost to accuracy at least when compared to the Black-Scholes model.

Figure 1 shows the VG fit and Figure 2 shows the Black-Scholes fit both for FRFT and FFT, for in-sample prices. Figure 3 and Figure 4 show the corresponding fits for out-ofsample prices. All figures show that both FRFT and FFT approaches yield very similar plots thus graphically illustrating that both models lead to much the same calibration fits. 
[FIGURE 1 APPROXIMATELY HERE]

[FIGURE 2 APPROXIMATELY HERE]

[FIGURE 3 APPROXIMATELY HERE]

[FIGURE 4 APPROXIMATELY HERE]

\subsection{Cross-Maturity and Cross-Strike Investigation}

We now investigate the pricing errors for four model specifications BS(FRFT), BS(FFT), VG (FRFT) and VG (FFT), across maturity and strike, relative to the closed-form BlackScholes prices. Our motivation is to examine the impact of the FRFT and FFT valuation methods and the impact of the underlying models (BS vs. VG) on the option price.

To reveal cross-strike features of FRFT and FFT under the time changed and original processes we express pricing errors as functions of strikes only, holding the maturity constant. We consider three different maturities observed in the market: minimum, mean and maximum corresponding to short, medium and long term options respectively:

$$
\operatorname{ERROR}^{\text {model }}\left(K_{i}\right)=P^{\text {model }}\left(K_{i}, t\right)-P^{B S}\left(K_{i}, t\right)
$$

Similarly, to reveal cross-maturity features of FRFT and FFT we express pricing errors as function of maturities only, holding the strike constant. Three different strikes are considered: minimum, equal to asset and maximum of the observed strikes in the market; these correspond to ITM, ATM and OTM options respectively: 


$$
\operatorname{ERROR}^{\operatorname{model}}\left(t_{i}\right)=P^{\operatorname{model}}\left(K, t_{i}\right)-P^{B S}\left(K, t_{i}\right)
$$

We plot cross-strike (left) and cross-maturity (right) errors, for different specifications, in Figure 5 using illustrative market data for the last week of July 2007. Figure 6 plots error surfaces across all ranges of strikes and maturities.

Three points stand out from these Figures. First, there is relatively little difference between the FRFT and FFT estimates of the BS option prices. Second, FRFT and FFT estimates of the VG option prices are often similar in pattern, but there are some notable differences; moreover, the differences between FRFT and FFT estimates of VG option prices seem to become more pronounced as we move down Figure 5 toward longer-term options (on the left hand side) and OTM options (on the right). Third, there are (as we would expect) pronounced differences between the BS and VG option prices. Put another way, if the VG model is correct then the BS model will often give poor valuations: these pricing errors are related (in complicated ways) to the options' maturities and strikes or moneyness.

\section{[FIGURE 5 APPROXIMATELY HERE]}

[FIGURE 6 APPROXIMATELY HERE]

\section{Conclusion}

In this study we have demonstrated how the standard Lévy-Kintchine formula can be interpreted as a series of shocks superimposed on a normal distribution, and how it can be 
used to value options using an illustrative example of a Variance-Gamma process. Using this derivation we have also been able to offer a correct solution to the mis-specification in the Lévy measure for the VG model derived by Geman (2002). We also calibrated the VG and BS models using weekly options data using both FFT and FRFT methods. We found that the FRFT is much faster than the FFT approach, economizing on $97-98 \%$ of the calculation time at the cost of small pricing errors; we also found that there are important differences between BS and VG option values, implying that the inappropriate use of the BS in a context where the true process was VG can lead to major pricing errors. These findings have important implications for the calibration of options models and for options risk management in general. This said, our work is limited in so far as we have only considered the application of the Lévy-Kintchine formula to a relatively simple VG process. Its application to more complex Lévy processes remains a topic for future study. 


\section{References}

Applebaum D (2004). Lévy Processes and Stochastic Calculus. Cambridge University Press, Cambridge

Bailey D and Swartztrauber P (1991). The fractional Fourier transform and applications, SIAM Review, 33, 389-404.

Black F and Scholes M (1973). The pricing of options and corporate liabilities, Journal of Political Economy, 81, 637-659.

Carr P and Madan D (1999). Option valuation using the fast Fourier transform, Journal of Computational Finance, 2, 61-73.

Carr P and Wu L (2004). Time-changed Lévy processes and option pricing, Journal of Financial Economics, 71, 113-141.

Chourdakis K M (2005). Option Pricing using the fractional FFT, Journal of Computational Finance, 8, 1-18.

Cont R and Tankov P (2004). Financial Modelling With Jump Processes, Chapman \& Hall/CRC Financial Mathematics Series.

Geman H, Madan D and Yor M (2001). Time changes for Lévy processes, Mathematical Finance, 11, 79-96.

Geman H (2002). Pure Jump Lévy processes for asset price modelling, Journal of Banking \& Finance, 26, 1297-1316.

Kyprianou A (2006). Introductory Lectures on Fluctuations of Lévy Processes with Applications, Springer.

Lee R W (2004). Option pricing by transform methods: extensions, unification, and error control, Journal of Computational Finance, 7, 51-86.

Madan D Carra P and Chang E (1998). The variance Gamma Process and option pricing model, European Finance Review, 2, 79-105.

Merton R C (1973) Theory of rational option pricing, Bell Journal of Economics and Management Science, 4, 141-183.

Sato Ken-Iti (1999). Lévy Processes and Infinitely Divisible Distributions, Cambridge University Press Cambridge

Schoutens W (2003). Lévy Processes in Finance: Pricing Financial Derivatives, John Wiley \& sons. Ltd. 
Shiryaev A N (1999). Essentials of Stochastic Finance: Facts, Models, Theory, World Scientific.

Watson G N (1944). A Treatise on the Theory of Bessel Functions, Cambridge University Press, Cambridge. 
Table 1: Calibration results under different specifications of Black-Scholes and Variance Gamma. We consider weekly traded options on S\&P500 from January 2007 to November 2007. The estimates reported are the averages of dynamic weekly calibrations over this sample period. The standard error of each estimate appears in parentheses. The average (over 44 weeks) weekly calibration time is also reported.

\begin{tabular}{|c|l|c|c|c|c|}
\hline \multicolumn{5}{|c|}{ Calibration Results } \\
\hline Specifications & RMSE & Average time (second) & $\sigma$ & $\theta$ & $\eta$ \\
\hline VG(FFT) & 2.6931 & 20.97 & 0.1294 & -0.1802 & 0.0786 \\
& & & $(0.0393)$ & $(0.0268)$ & $(0.0221)$ \\
VG(FRFT) & 2.7234 & 0.45 & 0.1232 & -0.1837 & 0.0839 \\
BS(FFT) & 3.1765 & 11.27 & $(0.0505)$ & $(0.0313)$ & $(0.0276)$ \\
& & & 0.1320 & & \\
BS(FRFT) & 3.2447 & 0.29 & $(0.0360)$ & & \\
& & & 0.1308 & & \\
BS(closed form) & 3.1764 & 0.063 & $(0.0362)$ & & \\
& & & 0.1320 & & \\
& & & $(0.0360)$ & & \\
& & & & \\
\end{tabular}


Figure 1: In-sample Variance Gamma fit under FFT(left) and FRFT(right). 0(market), *(model) and different colors are for different maturities as red(23dtm), blue(58dtm), green(86dtm), ceylon(149dtm), yellow(240dtm) and black(331dtm)
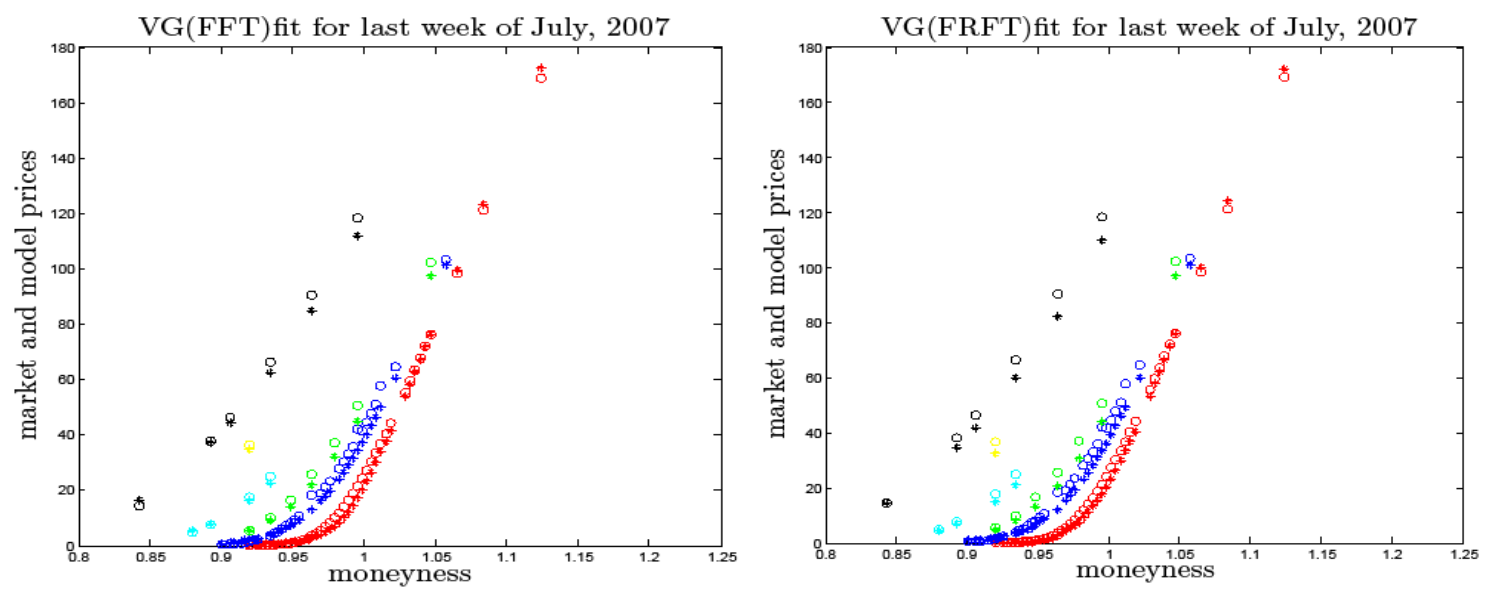
Figure 2: In-sample Black-Scholes fit under FFT (left) and FRFT (right). 0 (market), $*$ (model) and different colors are for different maturities as red (23dtm), blue (58dtm), green (86dtm), Ceylon (149dtm), yellow (240dtm) and black (331dtm)
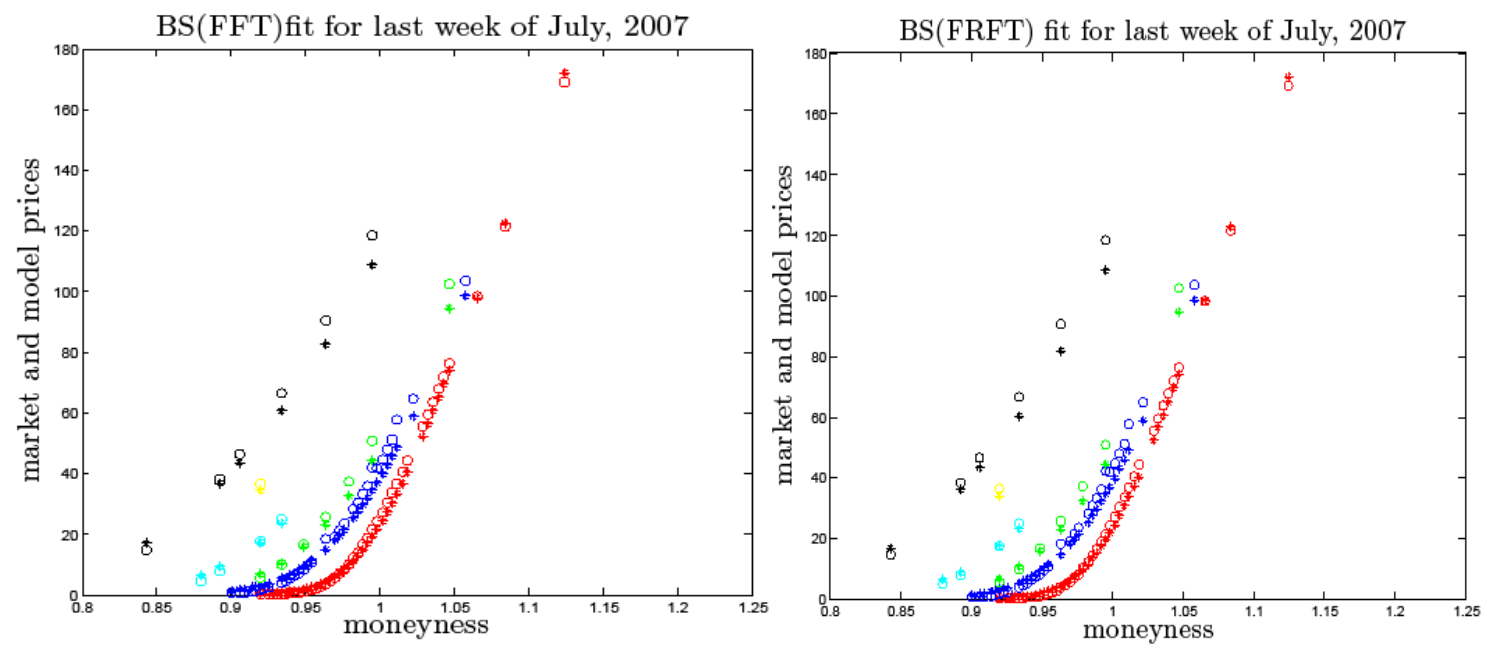
Figure 3: Out-of-sample Variance Gamma fit under FFT (left) and FRFT (right). 0 (market), *(model) and different colors are for different maturities as red (23dtm), blue (51dtm), green $(86 \mathrm{dtm})$, Ceylon $(114 \mathrm{dtm})$, yellow $(177 \mathrm{dtm})$, black (268dtm), magenta (359dtm)
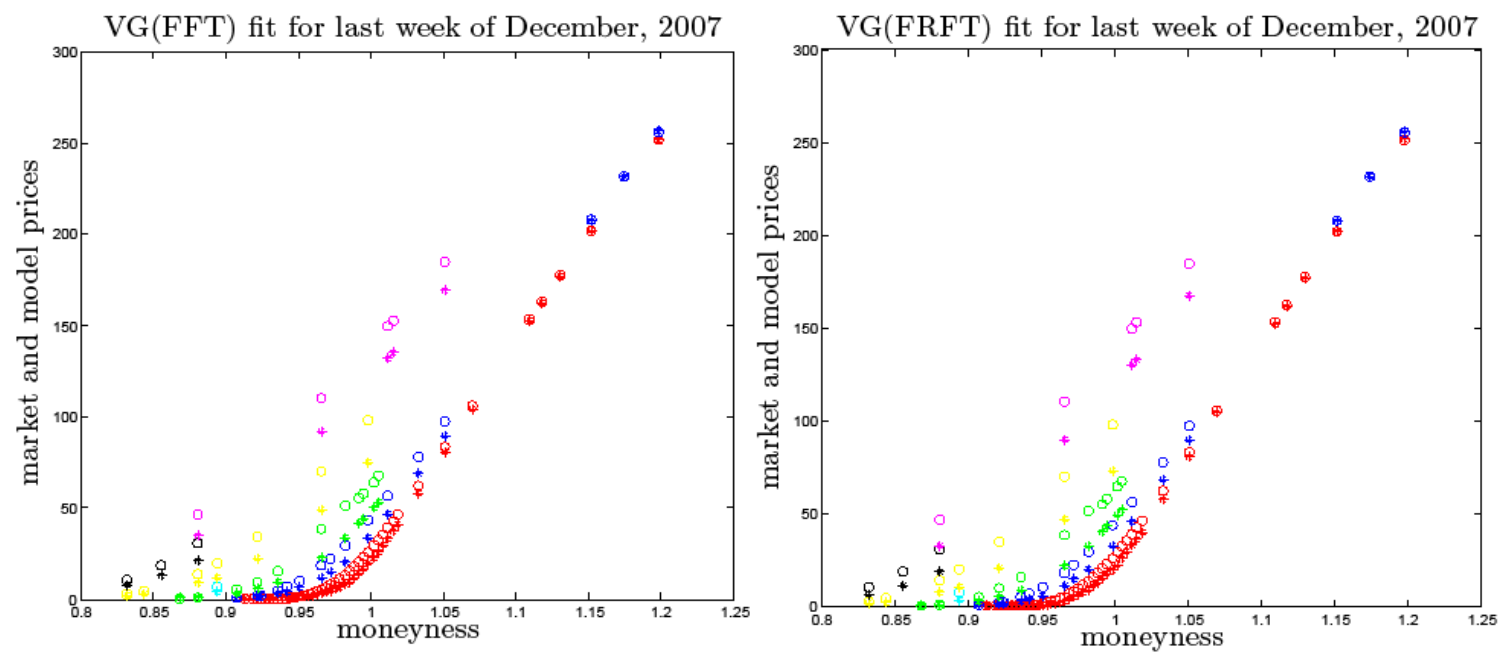
Figure 4: Out-of-sample Black-Scholes fit under FFT (left) and FRFT (right). 0 (market), $*$ (model) and different colours are for different maturities as red $(23 \mathrm{dtm})$, blue $(51 \mathrm{dtm})$, green (86dtm), Ceylon (114dtm), yellow (177dtm), black (268dtm), magenta (359dtm)
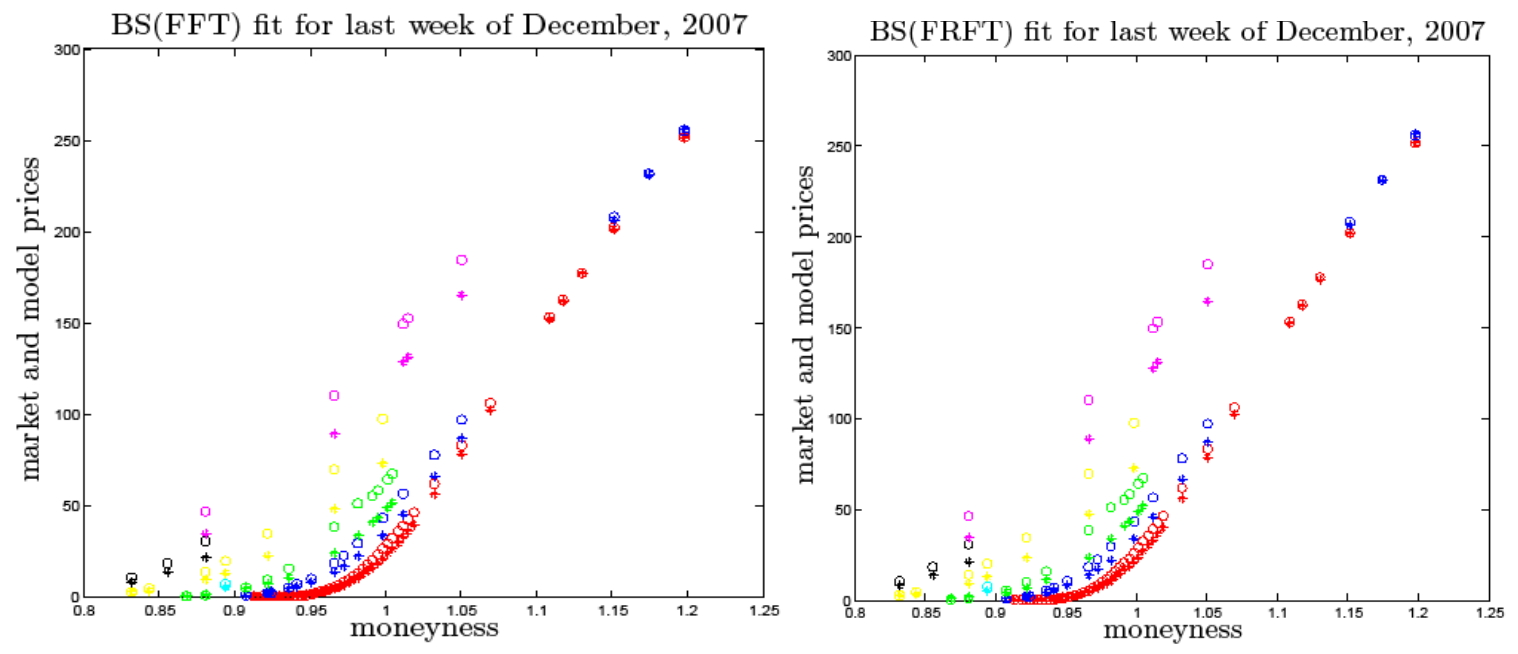
Figure 5: Cross strike (left) and cross-maturity (right) features of FRFT and FFT under Variance Gamma and Black-Scholes models, based on data for the last week of July 2007. Cross strike features are presented for short (top), medium (middle) and long (bottom) term options. Cross maturity features are presented for ITM (top), ATM (middle) and OTM (bottom) options. The average spot was 1518.09
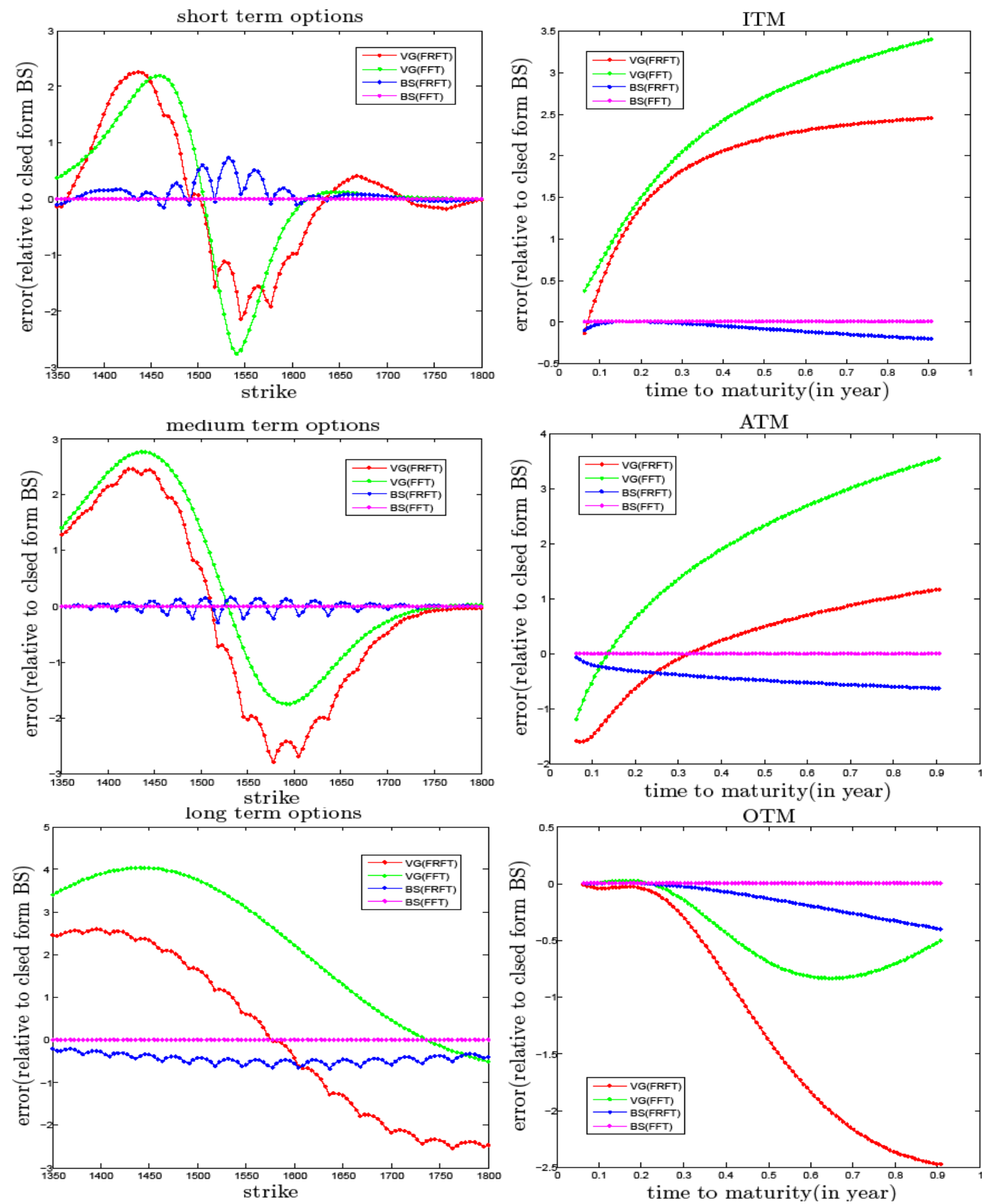
Figure 6: FRFT and FFT features under Variance Gamma and Black-Scholes models based on data for the last week of July 2007. The average spot was 1518.09

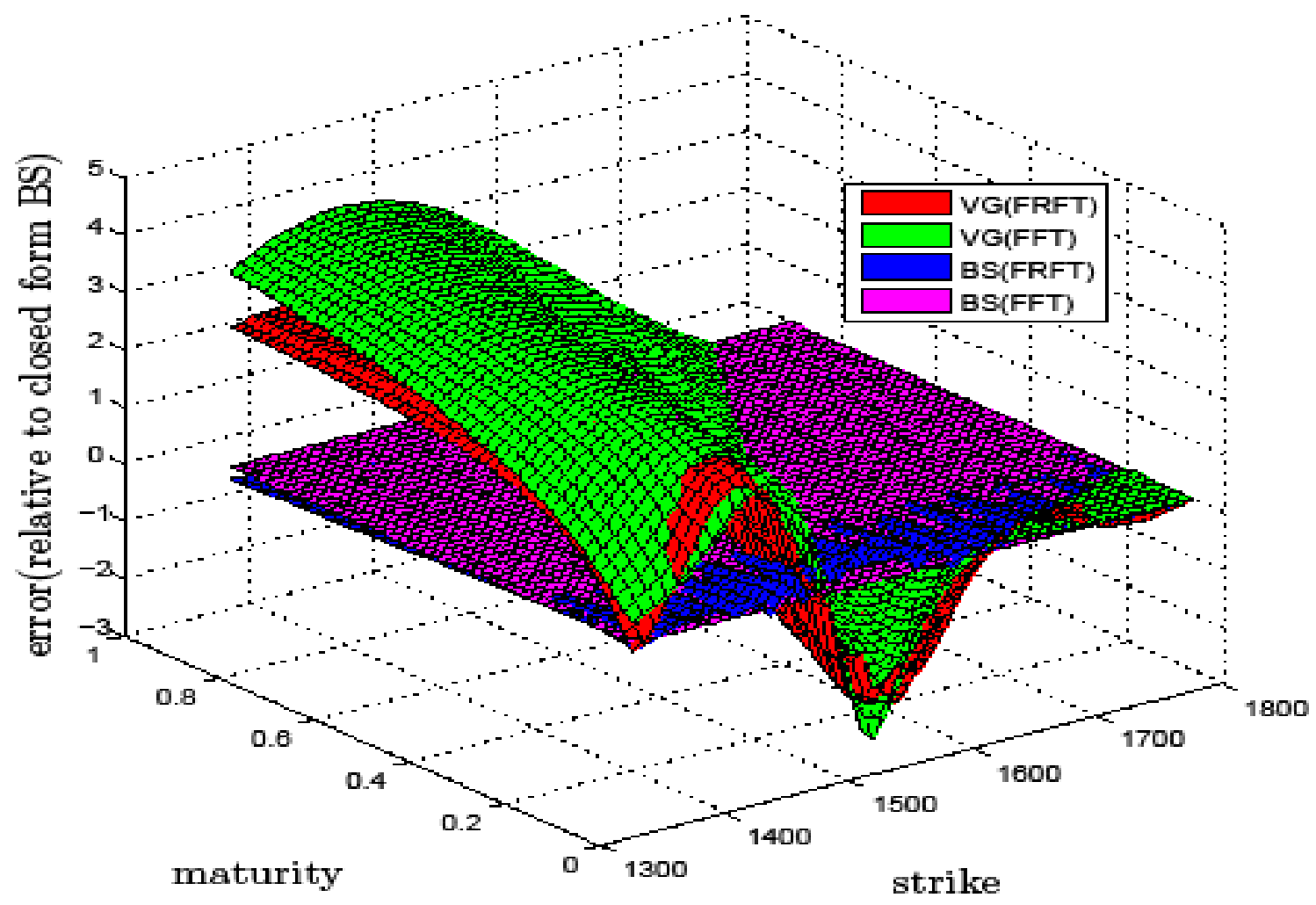

